\title{
CD1a and langerin: acting as more than Langerhans cell markers
}

\author{
Norikatsu Mizumoto and Akira Takashima
}

\author{
Department of Dermatology, University of Texas Southwestern Medical Center, Dallas, Texas, USA.
}

\begin{abstract}
Langerhans cells (LCs) represent a unique DC subset populating the outermost body surface, i.e., the epidermis. Although CD1a and langerin (CD207) are used as specific markers to distinguish LCs from other DC subsets, their immunological functions have remained mostly unknown. A new paper (see the related article beginning on page 701) demonstrates that LCs utilize these markers to induce cellular immune responses to Mycobacterium leprae: CD1a mediates the presentation of nonpeptide antigens to $T$ cells, while langerin facilitates uptake of microbial fragments and perhaps their delivery to a specialized subcellular compartment.
\end{abstract}

\section{Langerhans cell markers}

As a tissue constantly exposed to external insults, the skin is equipped with layers of protective mechanisms. Stratum corneum serves as a physical barrier against infectious microbes, foreign proteins, and harmful chemicals, and keratinocytes produce a wide array of pro- and anti-inflammatory cytokines, as well as antimicrobial peptides. As a skin resident member of the DC family of APCs, Langerhans cells (LCs) initiate both innate and adaptive immune responses to skin-relevant antigens, thereby acting as immunological sentinels. Although LCs were discovered in 1868 by gold chloride staining as a highly dendritic epidermal cell population, their lineage or function remained unknown until the 1960s and 1970s when appropriate LC "markers" were discovered. These include surface I-A molecules (i.e., murine MHC class II molecules; refs. 1 and 2), membrane-associated enzymatic activities to hydrolyze extracellular ATP (ecto-ATPase) (3), and rod/tennis racket-shaped intracellular organelles (Birbeck granules) (4). More specific markers, such as CD1a (5), E-cadherin (6), and langerin (7), are now known to distinguish LCs from other DC subsets.

Although identified initially as LC markers, these molecules have subsequently been shown to play functional roles in various

Nonstandard abbreviations used: carbohydrate recognition domain (CRD); Langerhans cell (LC); macrophage mannose receptor (MMR); MHC II-rich compartment (MIIC).

Conflict of interest: The authors have declared that no conflict of interest exists.

Citation for this article:

J. Clin. Invest. 113:658-660 (2004).

doi:10.1172/JCI200421140. aspects of LC biology. LCs employ I-A molecules for presentation of peptide antigens to CD4 T cells. CD39, which is responsible for LC-associated ecto-ATPase activities, regulates extracellular nucleotide-mediated skin inflammatory responses and immune reactions (8). Birbeck granules constitute a subdomain of the endosomal recycling compartment, perhaps being involved in an antigen loading process (9). E-cadherin-mediated LC-keratinocyte interaction facilitates epidermal retention of LCs (6) and also prevents their spontaneous maturation in the steady state (10). In this issue of the JCI, Hunger and colleagues have unveiled closely related functions of two additional LC markers: CD1a and langerin (11).

\section{LCs employ CD1a to present lipid antigens}

Group 1 CD1 isoforms (CD1a, CD1b, and CD1c in humans) expressed on APCs are capable of presenting various forms of selfand microbial lipid antigens to CD4, CD8, and double-negative $\mathrm{T}$ cell populations, whereas group 2 isoforms (CD1d in humans and mice) present an unusual glycosphingolipid, $\alpha$-galactosylceramide, to a specialized $\mathrm{T}$ cell subset known as NK T cells (reviewed in ref. 12). These findings have changed our classic view that $\mathrm{T}$ cells recognize only peptide antigens presented by MHC class I or class II molecules.

LCs express CD1a molecules at exceptionally high levels with virtually no detectable CD1b and only modest CD1c expression, whereas other DC subsets (e.g., dermal DCs and monocyte-derived DCs) predominantly display CD1b molecules with varying degrees of CD1a and CD1c expression. Following assembly with $\beta 2$-microglobulin in the endoplasmic reticulum, all CD1 isoforms are transported to the cell surface but are subsequently delivered to distinct endosomal and/or lysosomal compartments. For example, CD1b isoform is internalized in clathrincoated pits via a tyrosine-based motif in its cytoplasmic tail (YXXФ where Y is tyrosine, $\mathrm{X}$ is any amino acid, and $\Phi$ is a bulky hydrophobic residue), sorted to early endosomes, late endosomes/lysosomes, and MHC II-rich compartments (MIICs), and recycled back to the plasma membrane. By contrast, CD1a isoform, which lacks the YХХ $\Phi$ motif, mainly traffics between the cell surface and early recycling vesicles (13). CD1c and CD1d exhibit distinct trafficking patterns reflecting the difference in YXX $\Phi$ motifs and usages of adaptor-protein complexes (reviewed in ref. 12). The resulting diversity in surface expression profiles of CD1 isoforms among DC subsets and in their subcellular trafficking routes enables broad sampling of lipid antigens in different tissues and in various subcellular compartments.

Most of the lipid-reactive T cell clones generated from patients with Mycobacterium leprae or $M$. tuberculosis infection are restricted to the CD1b isoform. Hunger and colleagues reasoned that it might simply reflect the conventional use of monocyte-derived CD $1 b^{\text {high }}$ DC cultures as APCs for stimulating T cells. In fact, by using $\mathrm{CD} 34^{+}$progenitor-derived CD1a high DC cultures as APCs, they have generated several $M$. leprae-reactive, CD1arestricted double-negative $T$ cell clones from leprosy patients (11). Both CD $1 a^{\text {high }}$ DC cultures and LCs freshly procured from skin activated these $\mathrm{T}$ cell clones in the presence of $M$. leprae extracts, and anti-CD1a antibodies completely blocked this DC-T cell interaction. With respect to the identity of putative antigens, the CD1a-restricted $\mathrm{T}$ cell clones recognized non-peptide moieties in insoluble mycobacterial cell wall fractions but failed to respond to a tested panel of currently known CD1 lipid antigens, suggesting that one or more unusual (glyco)lipids are presented by CD1a molecules. These observations, together with recent other reports 

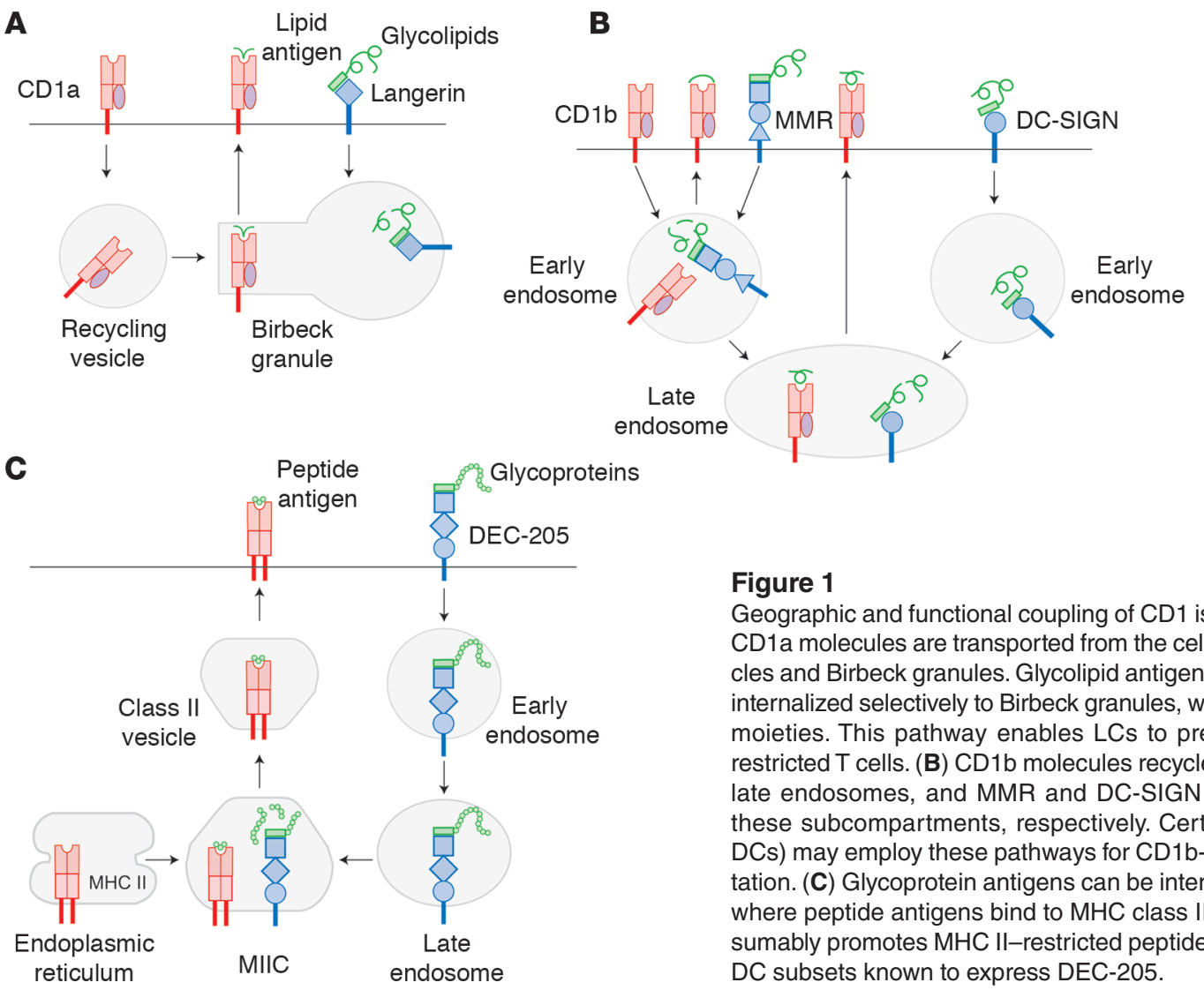

\begin{abstract}
Figure 1
Geographic and functional coupling of CD1 isoforms and C-type lectins. (A) CD1a molecules are transported from the cell surface to early recycling vesicles and Birbeck granules. Glycolipid antigens captured by langerin are also internalized selectively to Birbeck granules, where CD1a is coupled with lipid moieties. This pathway enables LCs to present lipid antigens to CD1arestricted T cells. (B) CD1b molecules recycle to early endosomes and then late endosomes, and MMR and DC-SIGN deliver glycolipid antigens to these subcompartments, respectively. Certain DC subsets (e.g., dermal DCs) may employ these pathways for CD1b-restricted lipid antigen presentation. (C) Glycoprotein antigens can be internalized via DEC-205 to MIICs, where peptide antigens bind to MHC class II molecules. This pathway presumably promotes MHC II-restricted peptide antigen presentation by many DC subsets known to express DEC-205.
\end{abstract}

$(14,15)$, now establish the concept that LCs employ CD1a to present mycobacterial lipid antigens to $\mathrm{T}$ cells.

\section{CD1a, langerin, and Birbeck granules: three components for orchestrating lipid antigen presentation by LCs}

Langerin (CD207) is a type II membrane-associated C-type lectin known to be expressed exclusively by LCs and to recognize mannose residues via its single carbohydrate recognition domain (CRD). Langerin is localized not only on the cell surface, but also intracellularly in close association with Birbeck granules. Transfection of langerin cDNA leads to Birbeck granule formation in fibroblasts. Upon binding to the cell surface, antilangerin antibodies are rapidly internalized and delivered to Birbeck granules. These observations indicate that langerin functions as an endocytic receptor for targeted transport of mannosecontaining ligands to Birbeck granules $(7,16)$.

Strikingly, Hunger and colleagues found that treatment of CD1 $1 \mathrm{a}^{\text {high }}$ DCs with antilangerin antibodies before, but not after, pulsing with $M$. leprae extracts completely abrogated their ability to stimulate CD1a-restricted T cell clones. Antilangerin antibodies also blocked the activation of CD1a-restricted $\mathrm{T}$ cell clones by freshly procured LCs (11). Importantly, antilangerin antibodies failed to inhibit the presentation of $M$. tuberculosis extracts by the same DC population to a CD1b-restricted T cell clone, with the implication that langerinmediated antigen uptake and delivery to Birbeck granules may be involved only in the CD1a-dependent antigen presentation pathway. In fact, intracellular CD1a molecules are found in close association with Birbeck granules $(13,15)$. Thus, it is tempting to speculate that Birbeck granules serve as a specialized subcompartment for orchestrating langerin-mediated, CD1a-dependent presentation of lipid antigens by LCs (Figure 1).

\section{C-type lectin receptors govern subcellular destinations of antigen delivery and antigen presentation pathways}

Langerin is not the only C-type lectin expressed by LCs or other DC subsets. DEC205 (CD205) is a type I membrane-associated C-type lectin containing ten contiguous CRDs and known to be expressed by various DC subsets (e.g., LCs, dermal DCs, and monocyte-derived DCs) and thymic epithelial cells (17). Macrophage mannose receptor (MMR) (CD206) is another type I lectin with eight CRDs and expressed by macrophages and select DC subsets including dermal DCs and monocyte-derived DCs, but not LCs (18). DC-SIGN (CD209) is a type II lectin with a single CRD and expressed by select DC subsets (again, excluding LCs) (19). Interestingly, epidermal LCs have been postulated to primarily employ langerin for binding HIV gp120, while dermal DCs mainly rely on MMR and DC-SIGN (20). Thus, different DC populations employ distinct sets of C-type lectins for capturing carbohydrate-bearing antigens (reviewed in ref. 21).

The above DC-associated C-type lectins further differ from each other in their subcellular sorting profiles. For example, DEC205 recycles into MIICs via a triacidic cluster in its cytoplasmic tail, thereby facilitating the formation of MHC II-peptide complexes. DEC-205-mediated antigen uptake and sorting, indeed, produced a 100-fold augmentation of CD4 $\mathrm{T}$ cell-stimulatory capacities $(17,22)$. Although MMR binds mannosylated antigens, this lectin receptor is constitutively internalized from the cell surface into early endosomes and recycled back to the surface. DC-SIGN, which also functions as an endocytic receptor and facilitates antigen presentation to T cells, shows a late endoso- 
mal trafficking profile (23). DEC-205, MMR, and DC-SIGN, but not langerin, contain the YXXФ motifs in cytoplasmic tails, suggesting that CD1 and C-type lectins share the same molecular sorting mechanisms for recycling to particular subcompartments (21).

In summary, each C-type lectin transports antigens to one or more specific subcompartments where selected antigen presentation molecules (i.e., MHC class I and II molecules and CD1 isoforms) recycle preferentially (Figure 1). Thus, C-type lectins govern not only the subcellular destination of newly captured antigens, but also their presentation pathway. In fact, geographic and functional coupling of $\mathrm{CD} 1 \mathrm{~b}$ and MMR has been reported to mediate CD1b-restricted presentation of mycobacterial lipoglycan antigens (24). The striking diversity in surface expression profiles of CD1 isoforms and C-type lectins among DC subsets and in intracellular trafficking routes of both classes of the receptors enables the DC system to survey a wide variety of tissues and subcompartments for carbohydrate-bearing antigens and to present distinct antigenic moieties to respective effector $\mathrm{T}$ cell populations.

Address correspondence to: Akira Takashima, Department of Dermatology, University of Texas Southwestern Medical Center, 5323 Harry Hines Boulevard, Dallas, Texas 75235, USA. Phone: (214) 648-3419; Fax: (214) 648-3472; E-mail: Akira.Takashima@ UTSouthwestern.edu.
1. Rowden, G., Lewis, M.G., and Sullivan, A.K. 1977. Ia antigen expression on human epidermal Langerhans cells. Nature. 268:247-248.

2. Klareskog, L., Tjernlund, U., Forsum, U., and Peterson, P.A. 1977. Epidermal Langerhans cells express Ia antigens. Nature. 268:248-250.

3. Wolff, K., and Winkelmann, R.K. 1967. Ultrastructural localization of nucleoside triphosphatase in Langerhans cells. J. Invest. Dermatol. 48:50-54.

4. Birbeck, M.S., Breathnach, A.S., and Everall, J.D. 1961. An electron microscope study of basal melanocytes and high-level clear cells (Langerhans cells) in vitiligo. J. Invest. Dermatol. 37:51-64.

5. Fithian, E., et al. 1981. Reactivity of Langerhans cells with hybridoma antibody. Proc. Natl. Acad. Sci. U. S. A. 78:2541-2544.

6. Tang, A., Amagai, M., Granger, L.G., Stanley, J.R., and Udey, M.C. 1993. Adhesion of epidermal Langerhans cells to keratinocytes mediated by E-cadherin. Nature. 361:82-85.

7. Valladeau, J., et al. 1999. The monoclonal antibody DCGM4 recognizes Langerin, a protein specific of Langerhans cells, and is rapidly internalized from the cell surface. Eur. J. Immunol. 29:2695-2704.

8. Mizumoto, N., et al. 2002. CD39 is the dominant Langerhans cell-associated ecto-NTPDase: modulatory roles in inflammation and immune responsiveness. Nat. Med. 8:358-365.

9. McDermott, R., et al. 2002. Birbeck granules are subdomains of endosomal recycling compartment in human epidermal Langerhans cells, which form where Langerin accumulates. Mol. Biol. Cell. 13:317-335.

10. Riedl, E., et al. 2000. Ligation of E-cadherin on in vitrogenerated immature Langerhans-type dendritic cells inhibits their maturation. Blood. 96:4276-4284.

11. Hunger, R.E., et al. 2004. Langerhans cells utilize CD1a and langerin to efficiently present nonpeptide antigens to T cells. J. Clin. Invest. 113:701-708. doi:10.1172/ JCI200419655.

12. Moody, D.B., and Porcelli, S.A. 2003. Intracellular pathways of CD1 antigen presentation. Nat. Rev. Immunol. 3:11-22.

13. Sugita, M., et al. 1999. Separate pathways for antigen presentation by CD1 molecules. Immunity. 11:743-752.
14. Pena-Cruz, V., Ito, S., Dascher, C.C., Brenner, M.B., and Sugita, M. 2003. Epidermal Langerhans cells efficiently mediate CD1a-dependent presentation of microbial lipid antigens to T cells. J. Invest. Dermatol. 121:517-521.

15. Salamero, J., et al. 2001. CD1a molecules traffic through the early recycling endosomal pathway in human Langerhans cells. J. Invest. Dermatol. 116:401-408.

16. Valladeau, J., et al. 2000. Langerin, a novel C-type lectin specific to Langerhans cells, is an endocytic receptor that induces the formation of Birbeck granules. Immunity. 12:71-81.

17. Jiang, W., et al. 1995. The receptor DEC-205 expressed by dendritic cells and thymic epithelial cells is involved in antigen processing. Nature. 375:151-155.

18. Sallusto, F., Cella, M., Danieli, C., and Lanzavecchia, A. 1995. Dendritic cells use macropinocytosis and the mannose receptor to concentrate macromolecules in the major histocompatibility complex class II compartment: Downregulation by cytokines and bacterial products. J. Exp. Med. 182:389-400.

19. Geijtenbeek, T.B., et al. 2000. Identification of DC-SIGN, a novel dendritic cell-specific ICAM-3 receptor that supports primary immune responses. Cell. 100:575-585.

20. Turville, S.G., et al. 2002. Diversity of receptors binding HIV on dendritic cell subsets. Nat. Immunol. 3:975-983.

21. Figdor, C.G., van Kooyk, Y., and Adema, G.J. 2002. $C$-type lectin receptors on dendritic cells and Langerhans cells. Nat. Rev. Immunol. 2:77-84.

22. Mahnke, K., et al. 2000. The dendritic cell receptor for endocytosis, DEC-205, can recycle and enhance antigen presentation via major histocompatibility complex class II-positive lysosomal compartments. J. Cell Biol. 151:673-684.

23. Engering, A., et al. 2002. The dendritic cell-specific adhesion receptor DC-SIGN internalizes antigen for presentation to T cells. J. Immunol. 168:2118-2126.

24. Prigozy, T.I., et al. 1997. The mannose receptor delivers lipoglycan antigens to endosomes for presentation to $\mathrm{T}$ cells by $\mathrm{CD} 1 \mathrm{~b}$ molecules. Immunity. 6:187-197.

\title{
Viral immunosuppression: disabling the guards
}

\author{
Marco Colonna
}

Department of Pathology and Immunology, Washington University School of Medicine, St. Louis, Missouri, USA.

\begin{abstract}
When facing an immune response, viruses can either attempt to elude them or confront them. A new report demonstrates that a lymphocytic choriomeningitis virus (LCMV) strain can suppress immune responses by targeting both development and activation of DCs (see the related article beginning on page 737). Ironically, type I IFN released in response to LCMV infection contributes to the blockade of DC development. The discovery of these immunosuppressive mechanisms provides new perspectives for the therapy of chronic infections associated with immunosuppression.
\end{abstract}

Nonstandard abbreviations used: $\alpha$-dystroglycan $(\alpha-\mathrm{DG})$; Armstrong 53b (ARM); clone 13 (Cl 13); natural IFN-producing cell (IPC); lymphocytic choriomeningitis virus (LCMV).

Conflict of interest: The author has declared that no conflict of interest exists.

Citation for this article:

J. Clin. Invest. 113:660-662 (2004).

doi:10.1172/JCI200421166.
Viruses have evolved multiple strategies to counteract host immune responses. Noncytopathic lymphocytic choriomeningitis virus (LCMV) employs several of these strategies to successfully infect mice. Initial immunosurveillance of LCMV infection is mediated by CTLs. However, this response may lead to selection of LCMV variants that carry mutations in the relevant CTL epitopes and, therefore, can elude cytotoxic responses $(1,2)$. Antibody responses are also essential for long-term protection. However, LCMV variants can evade humoral responses with point mutations that encode novel amino acids distorting the envelope glycoprotein epitope recognized by neutralizing antibodies (3).

\section{DCs as targets of LCMV immunosuppression}

Remarkably, LCMV not only eludes specific immune surveillance, but can also actively suppress immune responses. How is this accomplished? In this issue of the JCI, Sevilla and colleagues elucidate the mechanism 